\title{
Meniscal Transplants and Scaffolds: A Systematic Review of the Literature
}

\author{
Sean Dangelmajer, $\mathrm{BA}^{1}$, Filippo Familiari, $\mathrm{MD}^{2}$, Roberto Simonetta, $\mathrm{MD}^{3}$, Mehmet Kaymakoglu, $\mathrm{MD}^{4}$, \\ and Gazi Huri, $\mathrm{MD}^{4}$ \\ ${ }^{1}$ Department of Orthopaedic Surgery, Stanford School of Medicine, Stanford, CA, USA; ${ }^{2}$ Department of Orthopaedic and Trauma Surgery, Magna Græcia University, \\ Catanzaro, Italy; ${ }^{3}$ Department of Orthopaedic and Trauma Surgery, C.O.T. Cure Ortopediche Traumatologiche, Messina, Italy; ${ }^{4}$ Department of Orthopaedic and \\ Traumatology, Hacettepe University, Ankara, Turkey
}

The reported incidence of meniscal tears is approximately 61 per 100,000. In instances where preservation of the native meniscus is no longer a feasible option, meniscal allograft transplantation (MAT) and implants or scaffolds may be considered. The goal of this review was to compare the success and failure rates of two techniques, MAT and meniscal scaffolds, and make an inference which treatment is more preferable at the present time and future. Studies that met inclusion criteria were assessed for technique used, type of transplant used, number of procedures included in the study, mean age of patients, mean follow-up time, number of failures, failure rate, and reported reoperation rate. Fifteen studies for the MAT group and 7 studies for the meniscal scaffold group were identified. In this selection of studies, the average failure rate in the MAT group was $18.7 \%$ and average reoperation rate was $31.3 \%$. The average failure rate in the meniscal scaffold group was 5.6\%, and average reoperation rate was $6.9 \%$. It appears that although MAT is associated with high reoperation and failure rates, the limited number of studies on both MAT and scaffolds and mainly short-term results of scaffold studies make it difficult to make an objective comparison.

Keywords: Knee, Meniscus, Transplant, Tissue scaffold

\section{What is already known:}

- The reported incidence of meniscal tears is approximately 61 per 100,000 . In the United States, roughly 850,000 meniscal procedures are performed each year.

- Treatment options for meniscal injuries range from nonsurgical interventions such as physical therapy to surgical interventions including meniscus repair, meniscectomy, meniscal allograft transplantation (MAT) or more recently the use of meniscal scaffolds.

Received October 1, 2016; Revised (1st) December 1, 2016;

(2nd) January 16, 2017; Accepted January 17, 2017

Correspondence to: Filippo Familiari, MD

Department of Orthopaedic and Trauma Surgery, Magna Græcia University, Viale Europa, (Località Germaneto), 88100 Catanzaro, Italy Tel: +39-0961-364-7352, Fax: +39-0961-364-7104

E-mail: filippofamiliari@gmail.com

This is an Open Access article distributed under the terms of the Creative Commons Attribution Non-Commercial License (http://creativecommons.org/licenses/by-nc/4.0/) which permits unrestricted non-commercial use, distribution, and reproduction in any medium, provided the original work is properly cited.

\section{What are the new findings:}

- The results suggest a high level of variability in failure and reoperation rates among cohorts, highlighting a degree of bias that may be partially accounted for by surgical technique, type of device or transplant used, mean age of patients, follow-up time and number of patients included in the study.

- Failure rates following meniscal scaffold placement were within range of the studies assessing MAT-suggesting similar functional and clinical benefits.

- The lack of independent studies evaluating the outcomes of meniscal scaffolds makes it difficult to assess long-term outcomes, and studies to date may contain various biases including conflict of interest.

\section{Introduction}

The menisci are fibrocartilaginous tissues surrounding the tibial plateau inside the knee joint. Menisci are responsible for absorbing 50\%-70\% of the load across their respective compartments and for increasing the tibiofemoral contact area two to threefold $^{1,2)}$. Meniscal tissue also serves to lubricate the knee joint, and 
contributes to proprioception $^{2-5)}$.

Meniscus tears are common injuries either resulting from acute knee injury or from long-standing degenerative processes ${ }^{6,7)}$. Treatment options for meniscal injuries range from nonsurgical interventions such as physical therapy, to surgical interventions including meniscus repair, meniscectomy, MAT or more recently the use of meniscal scaffolds ${ }^{8)}$. In instances where preservation is no longer a viable option, meniscal transplantation and implants or scaffolds may be considered to restore knee biomechanics, distribute the load across a larger contact area compared to total meniscectomy, and potentially delay the onset of osteoarthritis $(\mathrm{OA})^{9)}$.

The first MAT procedure was performed in $1984^{10)}$. Ideal candidates for MAT are young to middle-aged patients presenting with moderate to severe pain post-meniscectomy ${ }^{11}$. There are several types of allografts available when performing an MAT, including fresh-frozen meniscal allografts with or without bone plugs, nonirradiated or irradiated ${ }^{12,13)}$. Despite its efficacy, a recent study has encouraged the exploration of new preservation techniques aimed at decreasing the apoptosis-mediated cell loss that occurs in cryopreservation ${ }^{14)}$. Furthermore, allograft size matching has been shown to be an important factor in functional outcomes and in preventing extrusion of the allograft ${ }^{15)}$. Surgical techniques include open versus minimally invasive arthroscopy, single tibial tunnel arthroscopy, keyhole, onlay, and bone plugs versus no bone plugs ${ }^{16-29)}$.

Unlike meniscal allograft transplantation, meniscal scaffolds can be performed in patients with partially resected menisci. Currently, there are two commercially available scaffolds; Collagen Meniscal Implant (CMI, Ivy Sports Medicine, Grafelfing, Germany) and polymer scaffold (Actifit, Orteq Bioengineering, London, UK). CMI is composed of type I collagen of the Achilles tendon. It was developed in the 1990s and marketed as trade name MenaFlex (ReGen Biologics, Hackensack, NJ, USA). It is designed for patients who have lost more than $50 \%$ of the meniscus but not all; therefore, it requires an outer rim and attachment to the anterior or posterior horn of the meniscus ${ }^{30)}$. CMI is only currently available in Europe due to political and regulatory process approval controversies. Actifit is composed of porous and acellular polyurethane segments. Produced in the 2000s, this scaffold aims to provide a template for tissue in-growth rather than a mechanical support. It degrades after a period of 5 years with the mechanical breakdown of urethane segments and macrophage phagocytosis ${ }^{31)}$. Integration of the implant was shown at second look arthroscopy after 12 months, and immature meniscus-like characteristics were apparent in histologic examination, with predominantly type-I collagen present ${ }^{32)}$.

The goal of this review was to answer the question: which of the meniscal treatment strategies promises more success in the meniscal treatment algorithm; meniscal transplantation or meniscal scaffolds? In this review, we explore common techniques, types of allografts or scaffolds used, and clinical outcomes such as failure and reoperation rates for MAT and meniscal scaffolds.

\section{Methods}

A systematic review was conducted according to Preferred Reporting Items for Systematic reviews and Meta-Analyses (PRISMA) guidelines using a PRISMA checklist ${ }^{33)}$. Two reviewers (SD and $\mathrm{MK}$ ) independently conducted the search using the MEDLINE/PubMed and Excerpta Medica/EMBASE databases. These databases were queried with the terms 'meniscal OR meniscus AND allograft AND transplant'. Search criteria for inclusion in the meniscal transplantation group included studies in English, published since 1984 (the date of the first meniscal allograft transplantation) until January 15, 2016 in human subjects. A total of 293 eligible results were found and subsequently assessed for studies including patients undergoing MAT, with documented complication, technique used, graft type, failure or reoperation rates. Failure was defined as poor postoperative knee function determined by moderate or severe pain with score systems such as Hospital Special Surgery. Exclusion criteria for the meniscal transplantation group included non-English studies, and studies exploring MAT in the context of other comorbidities such as failed tibial plateau fractures or replacement.

For the meniscal scaffold group, both databases were queried with the terms 'meniscal scaffold OR meniscus scaffold OR collagen meniscal implant'. A total of 220 eligible results were found and subsequently assessed for studies including documented complication, failure or reoperation rates. Failure was defined as poor knee function with severe persistent pain in selected studies. Search criteria for inclusion in the meniscal scaffold group included human subjects undergoing meniscal scaffold operation, studies in English, published since 2000 until January 15, 2016, with full text available, in human subjects. Studies assessing outcomes following transplant or scaffolding revisions were excluded from our analysis. A summary flowchart of our literature search can be found in Fig. 1. The quality of the evidence was classified using the US Preventive Services Task Force system for ranking levels of evidence. 


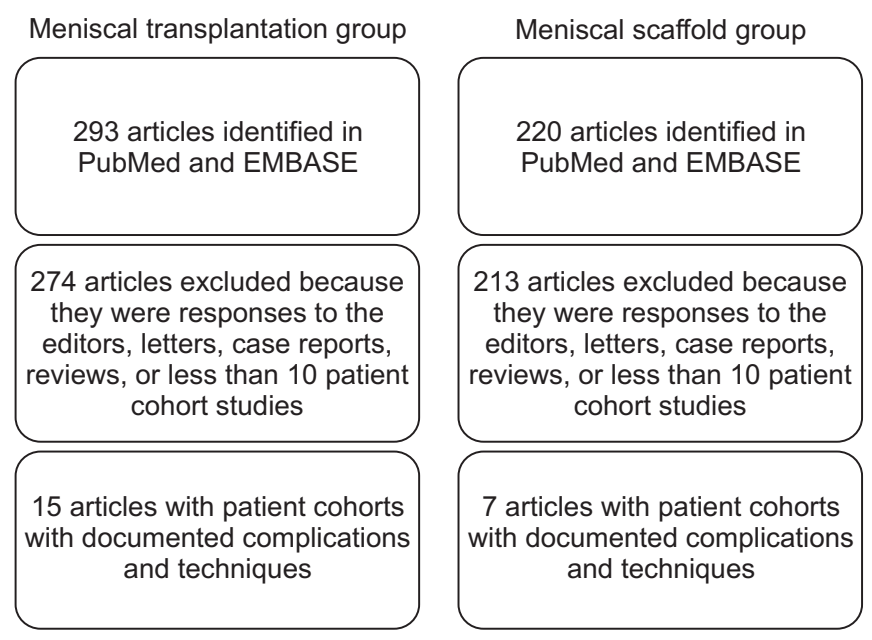

Fig. 1. Summary of the literature search for the meniscal transplantation and scaffold groups.

\section{Results}

\section{Meniscal Allograft Transplantation}

Studies were evaluated for techniques used, type of transplant, number of patients, patients' lost to follow-up, failure of meniscal allografts and reoperation rates. A summary of the review findings is included in Table 1.

The main type of meniscal allograft used was fresh-frozen cryopreserved allografts. Techniques varied, including double bone plug, minimally invasive arthroscopy, onlay, sutures only, bony fixation, open surgery and anchoring of horns with or without tunnels ${ }^{15-24,34)}$.

\section{1) Complication or failure rates}

Failure rates varied from $0 \%$ to $33.3 \%$ with patients undergoing fresh-frozen allograft transplantation with fixation through bone tunnels in the setting of patients with International Cartilage Repair Society (ICRS) chondral grade $3 \mathrm{~b}$ involving $>1 \mathrm{~cm}^{2}$ or worse presenting with the worst outcomes ${ }^{22,25)}$. High rates of allograft survival were reported in several studies with Rue et al. ${ }^{25)}$ reporting an overall failure rate of MAT in only $6.5 \%$ patients, indicating a 93.5\% MAT survival rate at a mean follow-up of 37.2 months and McCormick et al. ${ }^{16)}$ reporting a 95\% allograft survival rate at a mean of 5 years. Two of thirty-two patients experienced graft failure requiring subsequent reoperation in patients with MAT without bone plugs and a 3-year minimum followup; thus, exhibiting a $93.75 \%$ graft success rate at a mean followup of 40.4 months. Additionally, similar outcomes were reported with an $89 \%$ allograft success rate at a mean follow-up of 24.9 months ${ }^{28)}$.
Despite several studies reporting low failure rates, several revealed high rates of complications (as high as 30\%-46\%) including tears requiring repair, allograft removal, as well as infections requiring antibiotic treatment and lavage ${ }^{16,18,19,21,22)}$.

Despite the success of allograft survival, high rates of reoperation and failure were observed in a 15-year follow-up study of MAT with or without osteotomy; twenty-four of the eighty-six patients experienced MAT failure, and a total of $45.3 \%$ patients either required reoperation for meniscal debridement or due to MAT failure ${ }^{18)}$. McCormick et al. ${ }^{16)}$ reported 55 of 172 patients (32\%) underwent reoperation with simple surgical debridement being the most common (59\% of patients undergoing reoperation $)^{24)}$. In this selection of studies, the mean age of patients included in the MAT group was 32.1 years, average failure rate was $18.7 \%$, and average reoperation rate was $31.3 \%$ (among studies in which reoperation rates were included).

\section{2) Overall results and effects on $O A$}

According to Kellgren-Lawrence classification, 58\% of patients reported no increase in OA, while $42 \%$ noted a slight to moderate increase in OA with a minimum follow-up between 5 and 15 years ${ }^{35)}$. Longitudinal survival analysis in one study concluded that approximately $70 \%$ of patients at ten years exhibited a beneficial effect ${ }^{27)}$.

\section{Meniscal Scaffolds}

Studies were evaluated for techniques used, type of transplant, number of patients, patients' loss to follow-up, failure of meniscal scaffold and reoperation rates. A summary of the review findings is included in Table $2^{36-42)}$.

\section{1) Failure rates}

No cases of scaffold separation or meniscal degeneration were recorded in one study, with $50 \%$ of patients being able to return to their sporting activities at the time of the two- year followup $^{36)}$. A failure rate of $17.3 \%$ was reported in a study assessing polyurethane scaffolds ${ }^{37)}$. Complications related to 24 meniscal implants in a two-year follow-up study had only 1 patient undergoing a subsequent arthroscopic debridement 6 months after lateral collagen meniscus implantation for knee pain and swelling ${ }^{37)}$. Only 1 of 62 patients (1.6\%) experienced implant failure, with 2 additional patients of the 62 underwent a subsequent operation for chronic synovitis and infection ${ }^{39)}$. Most studies only reported failure rates without reporting reoperation rates separately. However, studies in which reoperation rates were reported separately indicate that the reoperation rates ranged from $4.2 \%$ to $9.5 \%$. 


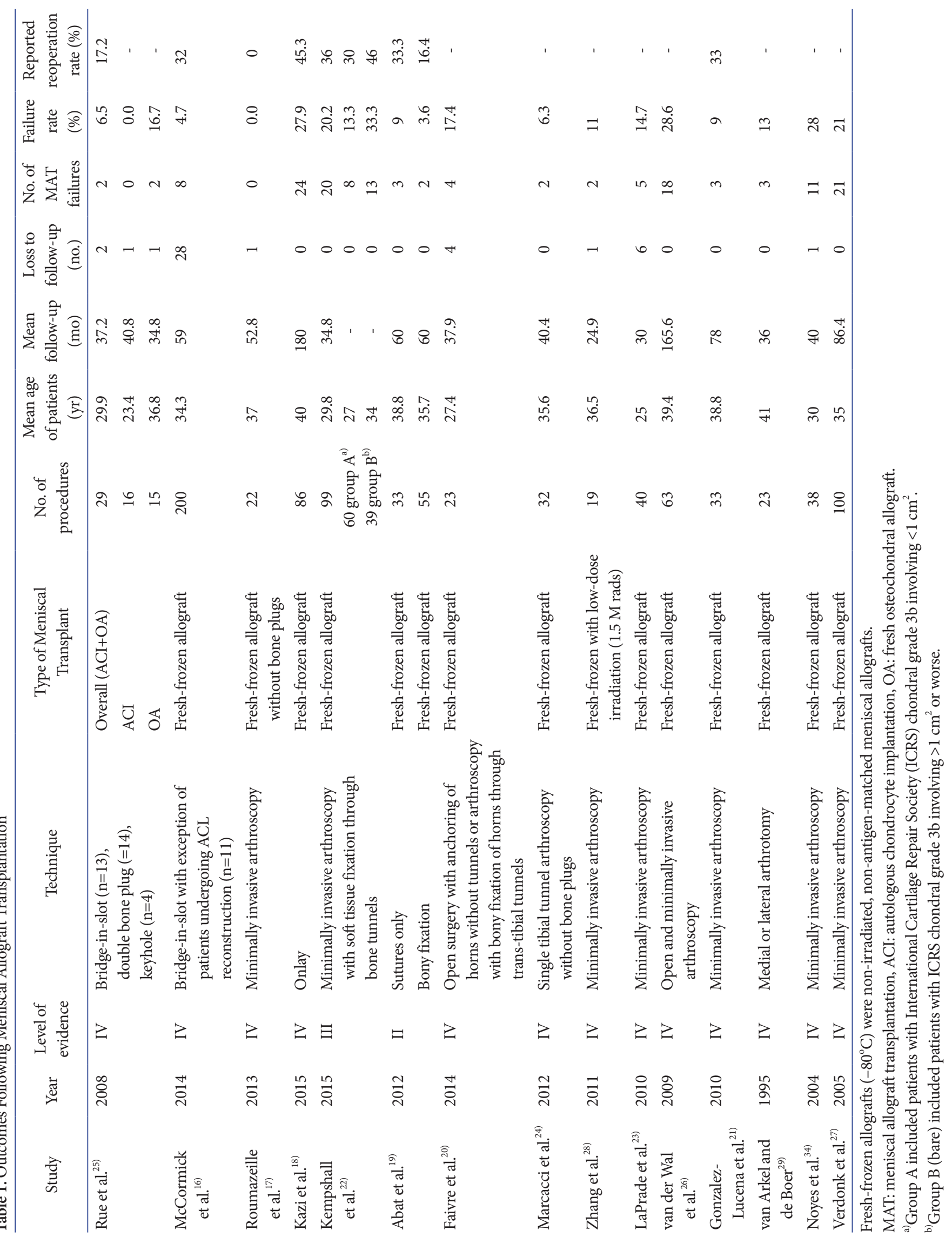




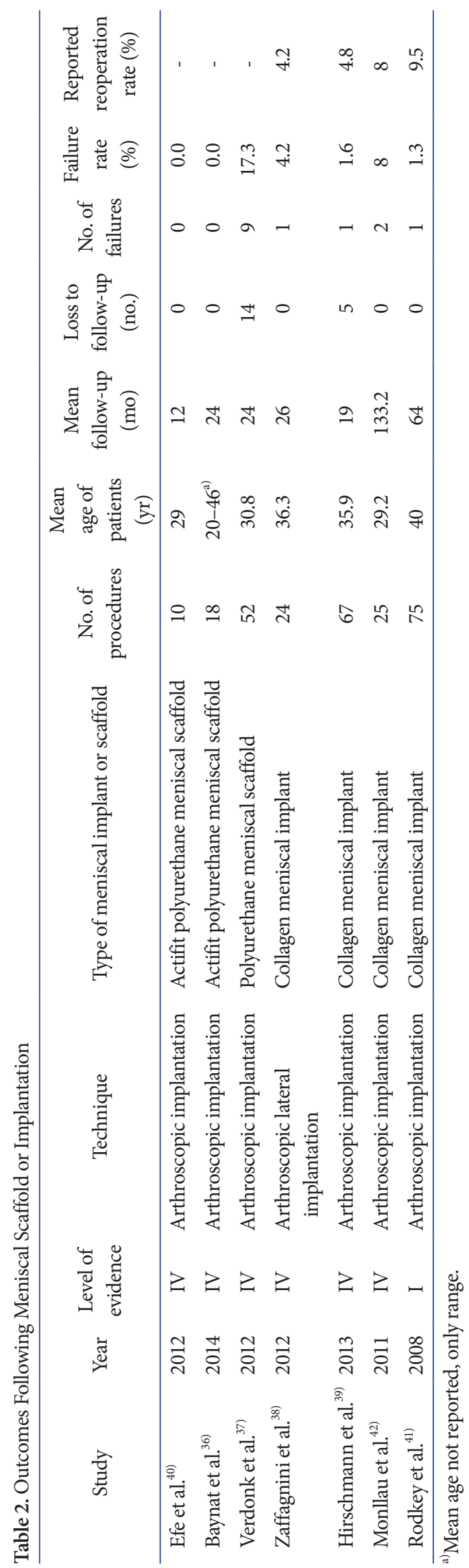

Among the largest study reporting reoperation rates, only 9.5\% of patients receiving collagen meniscal implants were subsequently reoperated on, with only 1 reported failure of implant in 75 patients with a mean follow-up of 64 months ${ }^{41}$.

2) Overall results

Among the studies included in the meniscal scaffold group, the mean age of patients was 35.7 years (excluding the study in which mean age was not included), average failure rate was $5.6 \%$, and average reoperation rate was $6.9 \%$ (among studies in which reoperation rates were included).

\section{Discussion}

MAT has been shown to provide improved clinical outcomes as well as delay the onset of $\mathrm{OA}$ in patients undergoing meniscectomy. Failure rates varied from $0 \%$ to $33.3 \%$ in the studies evaluated ${ }^{17,21)}$. Recent evaluation of MAT has indicated limited functional outcomes in patients returning to high-level physical activities. Its indication for the treatment of highly active asymptomatic meniscectomized patients such as athletes is less suitable. However, Noyes et al. ${ }^{34)}$ reported $76 \%$ of patients in their study returned to light low-impact sports (such as bicycling and swimming) without problems. Recently, Noyes et al. ${ }^{34)}$ also reported $63 \%$ survival of meniscal transplants in the same patient population. The latter study reveals that a decreased survival rate of $40 \%$ was observed at 15 years and the patients should be aware that a possible additional surgery can be needed at the end of this medium term. MAT failure due to extrusion appears to be correlated with the degree of articular cartilage wear, and patients with ICRS grades 3 and 4 experience up to 4.6-fold and 6.9-fold greater chance of extrusion, respectively ${ }^{43}$. Likewise, van Arkel et $\mathrm{al}^{44}$ indicated a $13 \%$ MAT failure rate, citing the failure was primarily due to malalignment resulting in impaired revascularization of the graft $^{29}$. Although extrusions are seen as a potential complication of MAT, the correlation with various clinical, radiologic, or arthroscopic outcomes is not entirely clear and further research must aim at clarifying its significance in evaluating clinical outcomes $^{45}$. That was the reason we excluded any studies that counted extrusion as a failure criteria.

Surgical technique also requires a total meniscectomy, and inherently, in the setting of partial meniscal resection, it is contraindicated. Postoperative magnetic resonance imaging (MRI) findings also do not correlate with second-look arthroscopy findings, making postoperative assessment more difficult ${ }^{46)}$. Nonetheless, many studies with symptomatic meniscectomy patients indicate clinical improvement in pain and daily life scores although the 


\section{Dangelmajer et al. Meniscal Transplants and Scaffolds: A Systematic Review}

average shrinkage in the size of the meniscus as shown on MRI is a concern ${ }^{47)}$. MAT was implicated as a good salvage therapy for the treatment of degenerative arthritis in post-meniscectomized knees ${ }^{26)}$. Evidence further suggests that immediate MAT versus delayed MAT (mean, 35 months; range, 9 to 92 months) is associated with less joint degeneration and muscle strength deficits ${ }^{48)}$.

There are few independent studies regarding the use of scaffolds. Most of the studies belong to the surgeons who have been involved in the development and design of the scaffolds themselves. It is also not well documented whether these meniscuslike scaffolds can transmit the load enough to prevent chondral wear. The main types of meniscal scaffold used were Actifit polyurethane meniscal scaffolds or $\mathrm{CMI}^{36,39-41,43,49)}$. A phase II feasibility study in eight patients showed new fibrocartilage matrix formation on biopsy tissue and integration into the scaffold was seen on second-look arthroscopy ${ }^{30)}$. Another clinical study showed that the scaffold is bio-resorbable with majority of the scaffold resorbed after 18 months ${ }^{45)}$. These studies are encouraging, but small control groups and short follow-up times call for additional research. Yet, pain relief and improved knee scores have been shown to be short-term advantages of meniscal scaffolds ${ }^{50)}$. More evidence is needed to detail the benefits that scaffolds could have over MAT, specifically in preventing chondral wear. With the advent of three-dimensional (3D) printing in recent years, new 3D imaging techniques make the future of meniscal scaffold design promising. In recent years, 3D printers and 3D imaging techniques have become more popular, and as such, personal 3D meniscal scaffolds are starting to be designed ${ }^{46)}$.

This study has inherent limitations including variability in follow-up time, which could skew the failure and reoperation rates, specifically in the studies where only short follow-up times were available. Moreover, several studies were excluded that focused on meniscal repairs in the setting of other concomitant procedures or revisions; all of which have beneficial insight into the treatment of meniscal injuries, yet were excluded in our assessment of the literature. Despite its limitations, this study provides a comprehensive overview of numerous level I-IV studies assessing complication rates, reoperation rates, techniques used, and number of failures following meniscal transplantation and scaffold procedures. The results of this study suggest that surgeons are performing MAT and meniscal scaffold placement in younger patients (mean age of 34.6 and 35.7, respectively).

The lack of independent studies evaluating the outcomes of meniscal scaffolds makes it difficult to assess long-term outcomes, and some studies to date may indicate bias. Additional prospective studies with larger control groups and more stan- dardized assessments of complications will be beneficial to improving long-term outcomes in young to middle-aged patients presenting with moderate to severe pain post-meniscectomy.

It appears that although MAT is associated with high reoperation and failure rates, the limited number of studies evaluating MAT and scaffolds makes it difficult to make an objective comparison.

\section{Conflict of Interest}

No potential conflict of interest relevant to this article was reported.

\section{References}

1. Verma NN, Kolb E, Cole BJ, Berkson MB, Garretson R, Farr J, Fregly B. The effects of medial meniscal transplantation techniques on intra-articular contact pressures. J Knee Surg. 2008;21:20-6.

2. Baratz ME, Fu FH, Mengato R. Meniscal tears: the effect of meniscectomy and of repair on intraarticular contact areas and stress in the human knee: a preliminary report. Am J Sports Med. 1986;14:270-5.

3. Thijs Y, Witvrouw E, Evens B, Coorevits P, Almqvist F, Verdonk R. A prospective study on knee proprioception after meniscal allograft transplantation. Scand J Med Sci Sports. 2007;17:223-9.

4. Jerosch J, Prymka M, Castro WH. Proprioception of knee joints with a lesion of the medial meniscus. Acta Orthop Belg. 1996;62:41-5.

5. Karahan M, Kocaoglu B, Cabukoglu C, Akgun U, Nuran R. Effect of partial medial meniscectomy on the proprioceptive function of the knee. Arch Orthop Trauma Surg. 2010;130:427-31.

6. Baker BE, Peckham AC, Pupparo F, Sanborn JC. Review of meniscal injury and associated sports. Am J Sports Med. 1985;13:1-4.

7. Englund M, Guermazi A, Gale D, Hunter DJ, Aliabadi P, Clancy M, Felson DT. Incidental meniscal findings on knee MRI in middle-aged and elderly persons. N Engl J Med. 2008;359:1108-15.

8. McCarty EC, Marx RG, DeHaven KE. Meniscus repair: considerations in treatment and update of clinical results. Clin Orthop Relat Res. 2002;(402):122-34.

9. Elattar M, Dhollander A, Verdonk R, Almqvist KF, Verdonk P. Twenty-six years of meniscal allograft transplantation: is 
it still experimental? A meta-analysis of 44 trials. Knee Surg Sports Traumatol Arthrosc. 2011;19:147-57.

10. Milachowski KA, Weismeier K, Wirth CJ. Homologous meniscus transplantation: experimental and clinical results. Int Orthop. 1989;13:1-11.

11. Verdonk R, Volpi P, Verdonk P, Van der Bracht H, Van Laer M, Almqvist KF, Vander Eecken S, Prospero E, Quaglia A. Indications and limits of meniscal allografts. Injury. 2013;44 Suppl 1:S21-7.

12. Jiang D, Zhao LH, Tian M, Zhang JY, Yu JK. Meniscus transplantation using treated xenogeneic meniscal tissue: viability and chondroprotection study in rabbits. Arthroscopy. 2012;28:1147-59.

13. Samitier G, Alentorn-Geli E, Taylor DC, Rill B, Lock T, Moutzouros V, Kolowich P. Meniscal allograft transplantation. Part 1: systematic review of graft biology, graft shrinkage, graft extrusion, graft sizing, and graft fixation. Knee Surg Sports Traumatol Arthrosc. 2015;23:310-22.

14. Villalba R, Pena J, Navarro P, Luque E, Jimena I, Romero A, Gomez Villagran JL. Cryopreservation increases apoptosis in human menisci. Knee Surg Sports Traumatol Arthrosc. 2012;20:298-303.

15. Jang SH, Kim JG, Ha JG, Shim JC. Reducing the size of the meniscal allograft decreases the percentage of extrusion after meniscal allograft transplantation. Arthroscopy. 2011;27:914-22.

16. McCormick F, Harris JD, Abrams GD, Hussey KE, Wilson H, Frank R, Gupta AK, Bach BR Jr, Cole BJ. Survival and reoperation rates after meniscal allograft transplantation: analysis of failures for 172 consecutive transplants at a minimum 2-year follow-up. Am J Sports Med. 2014;42:892-7.

17. Roumazeille T, Klouche S, Rousselin B, Bongiorno V, Graveleau N, Billot N, Hardy P. Arthroscopic meniscal allograft transplantation with two tibia tunnels without bone plugs: evaluation of healing on MR arthrography and functional outcomes. Knee Surg Sports Traumatol Arthrosc. 2015;23:264-9.

18. Kazi HA, Abdel-Rahman W, Brady PA, Cameron JC. Meniscal allograft with or without osteotomy: a 15-year follow-up study. Knee Surg Sports Traumatol Arthrosc. 2015;23:303-9.

19. Abat F, Gelber PE, Erquicia JI, Tey M, Gonzalez-Lucena G, Monllau JC. Prospective comparative study between two different fixation techniques in meniscal allograft transplantation. Knee Surg Sports Traumatol Arthrosc. 2013;21:1516-22.

20. Faivre B, Boisrenoult P, Lonjon G, Pujol N, Beaufils P. Lateral meniscus allograft transplantation: clinical and anatomic outcomes after arthroscopic implantation with tibial tunnels versus open implantation without tunnels. Orthop Traumatol Surg Res. 2014;100:297-302.

21. Gonzalez-Lucena G, Gelber PE, Pelfort X, Tey M, Monllau JC. Meniscal allograft transplantation without bone blocks: a 5- to 8-year follow-up of 33 patients. Arthroscopy. 2010;26:1633-40.

22. Kempshall PJ, Parkinson B, Thomas M, Robb C, Standell $\mathrm{H}$, Getgood A, Spalding T. Outcome of meniscal allograft transplantation related to articular cartilage status: advanced chondral damage should not be a contraindication. Knee Surg Sports Traumatol Arthrosc. 2015;23:280-9.

23. LaPrade RF, Wills NJ, Spiridonov SI, Perkinson S. A prospective outcomes study of meniscal allograft transplantation. Am J Sports Med. 2010;38:1804-12.

24. Marcacci M, Zaffagnini S, Marcheggiani Muccioli GM, Grassi A, Bonanzinga T, Nitri M, Bondi A, Molinari M, Rimondi E. Meniscal allograft transplantation without bone plugs: a 3-year minimum follow-up study. Am J Sports Med. 2012;40:395-403.

25. Rue JP, Yanke AB, Busam ML, McNickle AG, Cole BJ. Prospective evaluation of concurrent meniscus transplantation and articular cartilage repair: minimum 2-year follow-up. Am J Sports Med. 2008;36:1770-8.

26. van der Wal RJ, Thomassen BJ, van Arkel ER. Long-term clinical outcome of open meniscal allograft transplantation. Am J Sports Med. 2009;37:2134-9.

27. Verdonk PC, Demurie A, Almqvist KF, Veys EM, Verbruggen G, Verdonk R. Transplantation of viable meniscal allograft: survivorship analysis and clinical outcome of one hundred cases. J Bone Joint Surg Am. 2005;87:715-24.

28. Zhang H, Liu X, Wei Y, Hong L, Geng XS, Wang XS, Zhang J, Cheng KB, Feng H. Meniscal allograft transplantation in isolated and combined surgery. Knee Surg Sports Traumatol Arthrosc. 2012;20:281-9.

29. van Arkel ER, de Boer HH. Human meniscal transplantation: preliminary results at 2 to 5 -year follow-up. J Bone Joint Surg Br. 1995;77:589-95.

30. Rodkey WG, Steadman JR, Li ST. A clinical study of collagen meniscus implants to restore the injured meniscus. Clin Orthop Relat Res. 1999;(367 Suppl):S281-92.

31. Zuidema J, van Minnen B, Span MM, Hissink CE, van Kooten TG, Bos RR. In vitro degradation of a biodegradable polyurethane foam, based on 1,4-butanediisocyanate: a three-year study at physiological and elevated temperature. J Biomed Mater Res A. 2009;90:920-30. 
32. Verdonk R, Verdonk P, Huysse W, Forsyth R, Heinrichs EL. Tissue ingrowth after implantation of a novel, biodegradable polyurethane scaffold for treatment of partial meniscal lesions. Am J Sports Med. 2011;39:774-82.

33. Liberati A, Altman DG, Tetzlaff J, Mulrow C, Gøtzsche PC, Ioannidis JP, Clarke M, Devereaux PJ, Kleijnen J, Moher D. The PRISMA statement for reporting systematic reviews and meta-analyses of studies that evaluate health care interventions: explanation and elaboration. J Clin Epidemiol. 2009;62:e1-34.

34. Noyes FR, Barber-Westin SD, Rankin M. Meniscal transplantation in symptomatic patients less than fifty years old. J Bone Joint Surg Am. 2004;86:1392-404.

35. Vundelinckx B, Bellemans J, Vanlauwe J. Arthroscopically assisted meniscal allograft transplantation in the knee: a medium-term subjective, clinical, and radiographical outcome evaluation. Am J Sports Med. 2010;38:2240-7.

36. Baynat C, Andro C, Vincent JP, Schiele P, Buisson P, Dubrana F, Gunepin FX. Actifit synthetic meniscal substitute: experience with 18 patients in Brest, France. Orthop Traumatol Surg Res. 2014;100(8 Suppl):S385-9.

37. Verdonk P, Beaufils P, Bellemans J, Djian P, Heinrichs EL, Huysse W, Laprell H, Siebold R, Verdonk R; Actifit Study Group.. Successful treatment of painful irreparable partial meniscal defects with a polyurethane scaffold: two-year safety and clinical outcomes. Am J Sports Med. 2012;40:84453.

38. Zaffagnini S, Marcheggiani Muccioli GM, Bulgheroni $P$, Bulgheroni E, Grassi A, Bonanzinga T, Kon E, Filardo G, Busacca M, Marcacci M. Arthroscopic collagen meniscus implantation for partial lateral meniscal defects: a 2-year minimum follow-up study. Am J Sports Med. 2012;40:2281-8.

39. Hirschmann MT, Keller L, Hirschmann A, Schenk L, Berbig R, Lüthi U, Amsler F, Friederich NF, Arnold MP. One-year clinical and MR imaging outcome after partial meniscal replacement in stabilized knees using a collagen meniscus implant. Knee Surg Sports Traumatol Arthrosc. 2013;21:740-7.

40. Efe T, Getgood A, Schofer MD, Fuchs-Winkelmann S, Mann D, Paletta JR, Heyse TJ. The safety and short-term efficacy of a novel polyurethane meniscal scaffold for the treatment of segmental medial meniscus deficiency. Knee Surg Sports Traumatol Arthrosc. 2012;20:1822-30.

41. Rodkey WG, DeHaven KE, Montgomery WH 3rd, Baker CL Jr, Beck CL Jr, Hormel SE, Steadman JR, Cole BJ, Briggs KK. Comparison of the collagen meniscus implant with partial meniscectomy: a prospective randomized trial. J Bone Joint Surg Am. 2008;90:1413-26.

42. Monllau JC, Gelber PE, Abat F, Pelfort X, Abad R, Hinarejos $\mathrm{P}$, Tey M. Outcome after partial medial meniscus substitution with the collagen meniscal implant at a minimum of 10 years' follow-up. Arthroscopy. 2011;27:933-43.

43. Lee BS, Kim JM, Kim JM, Kim KA, Bin SI. Patient-related risk factors for the extrusion of lateral meniscal allograft transplants. Arthroscopy. 2015;31:699-706.

44. van Arkel ER, Goei R, de Ploeg I, de Boer HH. Meniscal allografts: evaluation with magnetic resonance imaging and correlation with arthroscopy. Arthroscopy. 2000;16:517-21.

45. Spencer SJ, Saithna A, Carmont MR, Dhillon MS, Thompson P, Spalding T. Meniscal scaffolds: early experience and review of the literature. Knee. 2012;19:760-5.

46. Moroni L, Lambers FM, Wilson W, van Donkelaar CC, de Wijn JR, Huiskesb R, van Blitterswijk CA. Finite element analysis of meniscal anatomical 3d scaffolds: implications for tissue engineering. Open Biomed Eng J. 2007;1:23-34.

47. Stollsteimer GT, Shelton WR, Dukes A, Bomboy AL. Meniscal allograft transplantation: a 1- to 5-year follow-up of 22 patients. Arthroscopy. 2000;16:343-7.

48. Jiang D, Ao YF, Gong X, Wang YJ, Zheng ZZ, Yu JK. Comparative study on immediate versus delayed meniscus allograft transplantation: 4- to 6-year follow-up. Am J Sports Med. 2014;42:2329-37.

49. Chalmers PN, Karas V, Sherman SL, Cole BJ. Return to high-level sport after meniscal allograft transplantation. Arthroscopy. 2013;29:539-44.

50. Zaffagnini S, Marcheggiani Muccioli GM, Lopomo N, Bruni D, Giordano G, Ravazzolo G, Molinari M, Marcacci M. Prospective long-term outcomes of the medial collagen meniscus implant versus partial medial meniscectomy: a minimum 10-year follow-up study. Am J Sports Med. 2011;39:977-85. 